\title{
Back to Original Frugality
}

\author{
RODRIGO A. VELEZ \\ Texas A\&M University
}

We review recent research on frugality of mechanisms for the procurement of a spanning network. Frugality here is defined as the ratio of the maximum price that can be charged to the buyer in some equilibrium to the true minimal cost. Previous negative results are qualified under natural restrictions in costs.

Categories and Subject Descriptors: J.4 [Social and Behavioral Sciences]: Economics

General Terms: Economics

Additional Key Words and Phrases: worst case scenario equilibrium analysis; frugality; minimum cost spanning tree problem; price of imperfect competition

\section{THE PROBLEM}

Consider the problem of a buyer who needs to procure a network spanning a set of three nodes. Let $\{e, f, g\}$ be the complete graph connecting these nodes. Assume that each edge can be exclusively constructed by a different seller, and thus, we can unambiguously refer to sellers $e, f$, and $g$. Let $c_{e}, c_{f}$, and $c_{g}$ be the sellers' costs of constructing the edges, and assume that $c_{e} \leq c_{f}<c_{g}$.

The buyer, with no access to the sellers' technology, and no knowledge of the true costs, will use a "mechanism" to procure the spanning network. Two popular options are the first price auction and the celebrated canonical VCG, or pivotal, mechanism.

In the first price auction the buyer asks the sellers for bids and selects a minimum cost spanning tree for the reported bids. Each selected seller constructs her edge and is paid her bid. In the pivotal mechanism sellers are asked to report their costs. Then, the buyer selects a minimum cost spanning tree for the reported costs. Each selected seller constructs her edge and is paid her reported cost plus the reduction in the cost of a minimum cost spanning tree due to the availability of the seller's edge at her reported cost.

If there is complete information among sellers, limit Nash equilibrium predicts that in the first price auction the efficient tree, i.e., $\{e, f\}$, is built and the buyer pays an amount $2 x$ where $x \in\left[c_{f}, c_{g}\right] .^{1,2}$ Thus, the maximum aggregate price paid

\footnotetext{
${ }^{1}$ Pure strategy Nash equilibria may fail to exist in this game depending on the rule that determines the winner of the auction when there are multiple minimal cost spanning trees at the reported bids.

${ }^{2}$ If edge $g$ is selected, seller $g$ must be paid at least $c_{g}$ and another edge, say $f$, is not selected. This cannot be true, for seller $f$ would be able to gain at least $c_{g}-c_{f}$ by bidding just below $c_{g}$. Thus, in equilibrium, seller $g$ bids some amount $x$ and sellers $e$ and $f$ bid just below $x$. Clearly, $x \geq c_{f}$, for seller $f$ can guarantee a non-negative payoff by bidding above $x$. Moreover, $x \leq c_{g}$, for otherwise seller $g$ would be able to gain by bidding just below $x$.
}

Author's address: rvelezca@econmail.tamu.edu 
by the buyer in a limit Nash equilibrium of the first price auction is $2 c_{g}$. One can easily see that this is also the payment of the buyer in the truthful equilibrium of the pivotal mechanism.

From the buyer's perspective it is relevant to compare the outcomes of a procurement mechanism with the ideal outcome in which the buyer has access to the sellers' technology. A handy statistic that provides such a comparison is the ratio of the highest predicted payment to the efficient cost [Archer and Tardos 2001; 2002]. In our example, this ratio for both the first price auction and the pivotal mechanism is $2 c_{g} /\left(c_{e}+c_{f}\right)$. Obviously, this ratio can be arbitrarily high if costs are unrestricted. However, it is at most 2 if costs are metric, i.e., if costs satisfy the familiar triangle inequality, which implies that $c_{g} \leq c_{e}+c_{f}$. This inequality would be satisfied whenever costs are a subadditive function of the "length" of an edge in a metric space.

In [Moulin and Velez 2013], we study the procurement games described above for an arbitrary number of nodes $n>2$. Surprisingly, the worst case scenario ratio of the maximum predicted payment to the efficient cost in the metric domain, for both first price auction and pivotal mechanism, is essentially that for the triangle, i.e., 2. The ratio increases swiftly when sellers can only bid for a subset of all edges, however.

\section{RESULTS}

More generally, we consider a buyer who procures a spanning network out of a set of edges that span $n>2$ nodes. We define the Price of Imperfect Competition (PIC) as the ratio of the maximum payment in a limit equilibrium of the first price auction, under complete information, to the efficient cost. (See Section 3 below for the relation of the PIC with the "frugality indices" defined by [Archer and Tardos 2001; 2002] and subsequent literature.) We assume that no seller has monopoly power, i.e., there is no seller who is the exclusive bidder for a cut of the graph of available edges. Our main results are:

(1) Each limit equilibrium of the first price auction is efficient. ${ }^{3}$

(2) Assume that costs are metric and each seller is the exclusive bidder for an edge.

(a) PIC is at most $n-1$.

(b) Assume that the graph of available edges is the complete graph.

i. PIC is at most and up to 2 if $n$ is odd, and is at most and up to $2 \frac{n-1}{n-2}$ if $n$ is even.

ii. Besides obtaining tightness of our bound, we characterize the trees that can be minimal cost spanning trees for some cost matrix that achieves the upper bound. Let $\gamma$ be spanning tree.

A. There is always a metric cost matrix for which $\gamma$ is a minimal cost spanning tree and for which PIC is 2.

B. Assume that $n$ is even. There is a metric cost matrix for which $\gamma$

\footnotetext{
${ }^{3}$ This is close to the efficiency of first price auction in matroid markets of [Chen and Karlin 2007], with the difference that we characterize unrefined limit equilibria and allow for each seller to be the exclusive bidder for multiple edges.
}

ACM SIGecom Exchanges, Vol. 12, No. 1, June 2013, Pages 49-52 
is a minimal cost spanning tree and for which PIC is $2 \frac{n-1}{n-2}$ if and only if $\gamma$ contains a perfect matching.

Surprisingly, worst case scenarios do not improve if costs are required to satisfy the stronger substitutability condition requiring that for each three edges forming a triangle $\{e, f, g\}, c_{e} \leq \max \left\{c_{f}, c_{g}\right\}$. Under this assumption, known as the ultrametric inequality, both 2.b.i and 2.b.iii above hold. However, in contrast to 2.b.ii, given a spanning tree $\gamma$, there is an ultrametric cost matrix for which $\gamma$ is a minimum cost spanning tree and for which PIC is 2, if and only if, $\gamma$ has at least a leaf edge of which the inner end node is of degree two.

Even though worst case PIC is the same in metric and ultrametric domains, intuitively PIC is lower on average for ultrametric costs. We confirm this in a simple probabilistic model.

(c) Assume that there is only one edge that is not available for purchase by the buyer. If $n=4$ or $n \geq 6$, then PIC is at most and up to 3 . If $n=5$, then PIC is at most and up to 4 .

(d) The worst case PIC increases swiftly when some edges are not available for purchase. We provide a lower bound, which we conjecture is tight, for the worst case PIC as a function of the number of available edges. If at most half of the edges are available for purchase our bound is tight: let $m \leq \frac{n(n-1)}{2}$; the worst case PIC among all metric cost problems in which $m$ edges are available for purchase is the maximum possible, i.e., $n-1$.

(e) If each seller is the exclusive bidder for exactly one edge, the maximum price charged to the buyer in a limit equilibrium of the first price auction is equal to the payment in the truthful equilibrium of the pivotal mechanism. (Thus, the PIC can be equally interpreted as measuring frugality of the pivotal mechanism.)

(3) Our structural results hold in the more general problem of procuring the basis of a matroid. First, limit Nash equilibria of the first price auction are efficient. Second, define the triangle inequality in a matroid as the requirement that no element of a circuit costs more than half the aggregate cost of the circuit. Then the PIC in the metric domain for a given matroid is bounded above by the dimension of the matroid. Our results illustrate that the whole range of values for the PIC in the metric domain can be achieved by minimum cost spanning tree problems.

\section{FRUGALITY}

Our work contributes to the literature concerned with the payment of a buyer who needs to procure a team to complete a complex task (see [Karlin et al. 2005] and references within). The main concerns in this literature have been (i) to define frugality indices that measure the extent to which the buyer overpays in a given situation; (ii) to evaluate the frugality of popular mechanisms; and (iii) to design mechanisms that minimize worst case scenario frugality indices [Archer and Tardos 2001; 2002; Talwar 2003; Elkind et al. 2004; Karlin et al. 2005; Chen and Karlin 2007] (see [Nisan et al. 2007, Section 13.5] for a survey). As in our work, the 
original frugality ratio was defined as the ratio of the highest predicted price to the efficient cost [Archer and Tardos 2001; 2002]. This ratio is a natural comparison between the strategic procurement situation and the best case situation for the buyer. Unfortunately, the first attempts to study it produced only negative results [Archer and Tardos 2001; 2002; Elkind et al. 2004]. This led to the belief that it was necessary to change the benchmark with respect to which the payment of the buyer is compared [Talwar 2003; Karlin et al. 2005]. The most prominent alternative, proposed by [Karlin et al. 2005], uses as benchmark cost the solution to a linear program that coincides with the pivotal payment in a matroid market. Thus, under this definition, the pivotal mechanism and the first price auction are the ideal of frugality in our environment.

Our work departs from this literature in an important way. We impose natural restrictions in the cost structure, while retaining the original benchmark cost with respect to which the buyer's payment is compared, i.e., the efficient cost. This allows us to obtain the first positive results for the unmodified frugality ratio for the popular first price auction and pivotal mechanisms. With this we open back the question of designing frugal mechanisms in restricted domains.

\section{REFERENCES}

Archer, A. And TArdos, E. 2001. Truthful mechanisms for one-parameter agents. In Proceedings, 42nd IEEE Symposium on the Foundations of Computer Science. 482-491.

Archer, A. And TARdos, E. 2002. Frugal path mechanisms. In Proceedings of the thirteenth annual ACM-SIAM symposium on Discrete algorithms. SODA '02. Society for Industrial and Applied Mathematics, Philadelphia, PA, USA, 991-999.

Chen, N. And Karlin, A. R. 2007. Cheap labor can be expensive. In Proceedings of the eighteenth annual ACM-SIAM symposium on Discrete algorithms. SODA '07. Society for Industrial and Applied Mathematics, Philadelphia, PA, USA, 707-715.

Elkind, E., Sahai, A., And Steiglitz, K. 2004. Frugality in path auctions. In Proceedings of the fifteenth annual ACM-SIAM symposium on Discrete algorithms. SODA '04. Society for Industrial and Applied Mathematics, Philadelphia, PA, USA, 701-709.

Karlin, A., Kempe, D., And Tamir, T. 2005. Beyond vcg: frugality of truthful mechanisms. In Foundations of Computer Science, 2005. FOCS 2005. 46th Annual IEEE Symposium on. 615-624.

Moulin, H. And Velez, R. A. 2013. The price of imperfect competition for a spanning network. Games Econ. Behav.. doi 10.1016/j.geb.2013.03.012.

Nisan, N., Roughgarden, T., Tardos, E., and Vazirani, V., Eds. 2007. Algorithmic Game Theory. Cambridge University Press, New York.

TALwAR, K. 2003. The price of truth: Frugality in truthful mechanisms. In STACS 2003, H. Alt and M. Habib, Eds. Lecture Notes in Computer Science, vol. 2607. Springer Berlin Heidelberg, $608-619$. 